July 2014

"On the Optimality of Pure Bundling for a Monopolist"

Domenico Menicucci, Sjaak Hurkens and Doh-Shin Jeon 


\title{
On the Optimality of Pure Bundling for a Monopolist*
}

\author{
Domenico Menicucci $^{\dagger} \quad$ Sjaak Hurkens ${ }^{\ddagger} \quad$ Doh-Shin Jeon ${ }^{\S}$
}

July 7,2014

\begin{abstract}
This paper considers a monopolist selling two objects to a single buyer with privately observed valuations. We prove that if each buyer's type has a non-negative virtual valuation for each object, then the optimal price schedule is such that the objects are sold only in a bundle; weaker conditions suffice if valuations are independently and identically distributed. Under somewhat stronger conditions, pure bundling is the optimal sale mechanism among all individually rational and incentive compatible mechanisms.
\end{abstract}

Keywords: Monopoly Pricing, Price discrimination, Multi-dimensional mechanism design, Pure Bundling.

JEL Codes: D42, D82, L11.

${ }^{*}$ We thank Mark Armstrong and Preston McAfee for helpful comments, and the participants of the presentations at the CEPR Conference on Applied IO 2013 (Bologna), Max-Planck Institute (Bonn), University of Cergy-Pontoise, University of Zurich.

†Universita’ degli Studi di Firenze, Italy; email: domenico.menicucci@unifi.it

${ }^{\ddagger}$ Institute for Economic Analysis (CSIC) and Barcelona GSE, Spain; email: sjaak.hurkens@gmail.com

$\S$ Toulouse School of Economics (IDEI, GREMAQ), France; email: dohshin.jeon@gmail.com 


\section{Introduction}

This paper studies the optimal sale mechanism for a monopolist which offers two different objects to a single buyer who privately observes her own valuations for the objects. ${ }^{1}$ We provide sufficient conditions under which the optimal (i.e., profit-maximizing) mechanism consists in pure bundling (i.e., selling both objects only in a bundle).

Since Myerson (1981) and Riley and Zeckhauser (1983), it is well-known that for the setting with a single object the optimal mechanism is such that the seller puts the object on sale at a suitable price, determined by the probability distribution of the buyer's valuation. However, the setting with two objects is considerably more difficult and Manelli and Vincent (2006, 2007, 2012) prove that the form of the optimal mechanism depends on the distribution of the buyer's valuations, unlike in the one-object environment. In some cases the optimal mechanism is characterized by a price schedule which specifies a price for each object and a price for the bundle of the two objects, but in other cases the optimal mechanism assigns the objects randomly. ${ }^{2}$ In general, many different mechanisms can be optimal as the distribution of valuations varies, and typically not much is known about the optimal mechanism for a given distribution, except for a few specific settings. For instance, when the valuations are independently and identically distributed, Manelli and Vincent (2006), Pavlov (2011), and Giannakopoulos (2014) solve the problem for uniform or exponential distributions; Hart and Nisan (2014) prove that pure bundling is optimal if the density for each valuation decreases quickly. Hart and Nisan (2014) also try to bound from below the fraction of the optimal profit that can be obtained by selling the objects separately (i.e., posting a suitable price for each object, as in two unrelated one-object settings), or by selling them in a bundle. For instance, they show that when valuations are i.i.d., separate sales yield at least 73 percent of the optimal profit. Conversely, when valuations are correlated, separate selling cannot guarantee any positive fraction of the optimal profit. ${ }^{3}$

This paper's main contribution consists in providing sufficient conditions which make it optimal to sell the objects as a bundle. Precisely, in Section 3, we assume that the seller

\footnotetext{
${ }^{1}$ We use "she" for the buyer and "he" for the seller.

${ }^{2}$ Hart and Reny (2013) prove that the seller's profit in the optimal mechanism may decrease when the buyer's valuations increase in the sense of first order stochastic dominance, another feature which does not arise in one-object setting.

${ }^{3} \mathrm{Li}$ and Yao (2013) improve some of the lower bounds considered in Hart and Nisan (2014).
} 
uses price schedules which specify a price for each object and a price for the bundle. In this setting we show that if the virtual valuation for each object is non-negative for each buyer type, then the optimal price schedule is such that each buyer type either buys the bundle, or buys nothing; weaker conditions suffice for this result if the valuations are independently and identically distributed. More precisely, we prove that for any mixed bundling price schedule where some types buy only one of the two objects, it is strictly profitable to reduce the price of the bundle. Such price change induces a fraction of types who buy at most one object to buy the bundle, which increases the profit because the virtual valuation of a single object is positive for any type. This result complements McAfee, McMillan, and Whinston (1989), who prove that selling the objects separately is suboptimal when valuations are independently distributed, as introducing a small discount for the bundle (mixed bundling) increases the profit. Under our sufficient conditions, mixed bundling is dominated by pure bundling.

Our framework can be applied to business-to-business transactions in which a given seller sells its objects to multiple buyers by applying a third-degree price discrimination: the seller can offer a different price schedule to each different buyer. In this situation, as long as buyers do not compete in the same market, each buyer can be treated as a separate market. The seller should have some precise (albeit imperfect) idea about each business customer's valuations for his objects. For instance, the seller can conduct market studies for this purpose. Our sufficient condition that the virtual valuation is positive for all types is likely to be met for serious buyers whose minimum valuations are high enough. Then, pure bundling is the optimal sale strategy. All other things being equal, pure bundling is more likely to be optimal for objects with lower costs of production implying that when licensing highly-valued patents, pure bundling is likely to be profit-maximizing.

Since mechanisms based on price schedules are only a subset of the set of all individually rational and incentive compatible mechanisms, in Section 4, we consider a setting in which the seller may use any mechanism in the set of individually rational and incentive compatible mechanisms. We give sufficient conditions for pure bundling to be optimal among all mechanisms in this set. These conditions are unrelated to those in Hart and Nisan (2014), and can in part be interpreted as a strengthening of the condition of non-negative virtual valuations. 


\section{The model}

A monopolist, henceforth denoted by M, owns two indivisible objects which are worthless to him, ${ }^{4}$ and faces a buyer interested in these objects. ${ }^{5} \mathrm{M}$ wishes to design a mechanism to maximize his expected profit (i.e., revenue) from trading with the buyer. The buyer is risk neutral and privately observes her own valuations for the objects, denoted with $v_{1}, v_{2}$. Her payoff from trading with the seller is given by her gross utility minus the payment to $\mathrm{M}$, and her gross utility is $v_{1}+v_{2}$ if she consumes both objects, is $v_{i}$ if she consumes only object $i$ (for $i=1,2$ ), is zero if she consumes nothing. The seller views $v_{i}$ as a realization of a random variable with a c.d.f. $F_{i}$ and a density $f_{i}$ which is continuous and strictly positive in the support $\left[\underline{v}_{i}, \bar{v}_{i}\right]$ satisfying $0<\underline{v}_{i}<\bar{v}_{i}$, for $i=1,2$. Moreover, the distributions of valuations are stochastically independent. Let $V \equiv\left[\underline{v}_{1}, \bar{v}_{1}\right] \times\left[\underline{v}_{2}, \bar{v}_{2}\right]$ denote the set of possible buyer types.

\section{Price schedules}

In this section we assume that $\mathrm{M}$ offers the objects to the buyer by posting a price schedule $\left(p_{1}, p_{2}, P\right)$ which specifies a price $p_{i}$ for good $i$, for $i=1,2$, and a price $P$ for the bundle of the two objects. Without loss of generality we consider $\left(p_{1}, p_{2}, P\right)$ satisfying $p_{1} \geq \underline{v}_{1}, p_{2} \geq \underline{v}_{2}$, $P \geq \underline{v}_{1}+\underline{v}_{2}$. After seeing $\left(p_{1}, p_{2}, P\right)$, the buyer chooses the alternative which maximizes her own payoff. Notice that for each type of buyer, the buyer's probability to obtain object $i$, or the bundle, is in $\{0,1\}$. For this reason this mechanism is said to be deterministic.

Let $S_{1}, S_{2}, S_{12}$ denote the set of types who, respectively, buy object 1 only, object 2 only, the bundle. Let $\mu_{1}, \mu_{2}, \mu_{12}$ denote the measure of $S_{1}, S_{2}, S_{12}$, respectively. In order to derive $\mu_{1}, \mu_{2}, \mu_{12}$ as a function of $\left(p_{1}, p_{2}, P\right)$, we need to distinguish the case of $P \leq p_{1}+p_{2}$ from the case of $P>p_{1}+p_{2} \cdot{ }^{6}$ In this section, we focus on the first case and consider the second case (for which we obtain the same results) in the appendix.

\footnotetext{
${ }^{4}$ Our results extend in a straightforward way to the case in which $\mathrm{M}$ has valuations for the objects or incurs production costs. See extensions in Section 3.

${ }^{5}$ Alternatively, we can assume that the monopolist sells the two objects to a continuum of buyers.

${ }^{6}$ Imposing the inequality $P \leq p_{1}+p_{2}$ makes sense if the seller is unable to monitor the buyer's purchases. That may be the case if the seller faces many anonymous buyers.
} 
Then, M's profit is given by:

$$
\pi=\mu_{1} p_{1}+\mu_{2} p_{2}+\mu_{12} P
$$

A type $\left(v_{1}, v_{2}\right)$ belongs to $S_{1}$ if and only if $v_{1} \geq p_{1}$ (i.e., buying only object 1 is better than buying nothing) and $v_{2}<P-p_{1}$ (i.e., buying only object 1 is better than buying the bundle). ${ }^{8}$ Hence

$$
\mu_{1}\left(p_{1}, p_{2}, P\right)=\left[1-F_{1}\left(p_{1}\right)\right] F_{2}\left(P-p_{1}\right) .
$$

Notice that if $p_{1}>\bar{v}_{1}$ and/or $p_{1}>P-\underline{v}_{2}$, then $S_{1}=\emptyset$ and $\mu_{1}=0$ since for each type, $v_{1}<p_{1}$ and/or $v_{2}>P-p_{1}$. However, $\pi$ remains unchanged if $\mathrm{M}$ lowers $p_{1}$ to satisfy $p_{1}=\min \left\{\bar{v}_{1}, P-\underline{v}_{2}\right\}$, since then still $\mu_{1}=0 .{ }^{9}$ Therefore, without loss of generality, we assume that $\mathrm{M}$ chooses $p_{1}$ satisfying $p_{1} \leq \min \left\{\bar{v}_{1}, P-\underline{v}_{2}\right\}$.

Likewise, $\left(v_{1}, v_{2}\right) \in S_{2}$ if and only if $v_{2} \geq p_{2}$ and $v_{1}<P-p_{2}$. Hence

$$
\mu_{2}\left(p_{1}, p_{2}, P\right)=\left[1-F_{2}\left(p_{2}\right)\right] F_{1}\left(P-p_{2}\right)
$$

and we assume without loss of generality that $\mathrm{M}$ chooses $p_{2}$ such that $p_{2} \leq \min \left\{\bar{v}_{2}, P-\underline{v}_{1}\right\} .{ }^{10}$

Finally, $\left(v_{1}, v_{2}\right) \in S_{12}$ if and only if $v_{1}+v_{2}-P \geq \max \left\{0, v_{1}-p_{1}, v_{2}-p_{2}\right\}$, which is equivalent to $v_{1}+v_{2} \geq P, v_{1} \geq P-p_{2}, v_{2} \geq P-p_{1}$ : see Figure $1($ a) below. Hence

$$
\mu_{12}\left(p_{1}, p_{2}, P\right)=\int_{P-p_{1}}^{p_{2}}\left[1-F_{1}\left(P-v_{2}\right)\right] f_{2}\left(v_{2}\right) d v_{2}+\left[1-F_{1}\left(P-p_{2}\right)\right]\left[1-F_{2}\left(p_{2}\right)\right] .
$$

We define a mixed bundling schedule and a pure bundling schedule as follows.

Definition 1 We say that $\left(p_{1}, p_{2}, P\right)$ is a mixed bundling schedule if $\mu_{1}\left(p_{1}, p_{2}, P\right)>0$ and/or $\mu_{2}\left(p_{1}, p_{2}, P\right)>0$; it is a pure bundling schedule if $\mu_{1}\left(p_{1}, p_{2}, P\right)=\mu_{2}\left(p_{1}, p_{2}, P\right)=0$.

In particular, $\left(p_{1}, p_{2}, P\right)$ is a pure bundling schedule if $P=\min \left\{p_{1}+\underline{v}_{2}, p_{2}+\underline{v}_{1}\right\}$.

\footnotetext{
${ }^{7}$ As a tie-breaking rule we assume that each buyer who is indifferent between two or more alternatives chooses the alternative which maximizes her gross utility. However, since the distribution of types is atomless, how indifferences are broken does not affect the results.

${ }^{8}$ These two inequalities, jointly with $P \leq p_{1}+p_{2}$, imply $v_{1}-p_{1} \geq 0>v_{2}-p_{2}$. Hence buying only object 1 is better than buying only object 2 .

${ }^{9}$ This reduction of $p_{1}$ does not affect neither $\mu_{2}$ nor $\mu_{12}$ since, given $p_{1} \geq \min \left\{\bar{v}_{1}, P-\underline{v}_{2}\right\}, p_{1}$ does not affect any type's preferred alternative among buying only object 2 , buying the bundle, and buying nothing.

${ }^{10}$ Notice that $p_{1} \leq \bar{v}_{1}, p_{2} \leq \bar{v}_{2}$ and $P \leq p_{1}+p_{2}$ imply $P-p_{1} \leq \bar{v}_{2}$ and $P-p_{2} \leq \bar{v}_{1}$.
} 
As a benchmark, consider a single-object monopolist facing a buyer whose valuation for the object has a c.d.f. $F$ and a density $f$ which is continuous and positive in the support $[\underline{v}, \bar{v}]$ satisfying $0<\underline{v}<\bar{v}$. Then it is well known that the profit-maximizing price is either $\underline{v}$, or solves the equation $J(x)=0$ with $J(x) \equiv x-\frac{1-F(x)}{f(x)}$ for $x \in[\underline{v}, \bar{v}]$. In particular, the optimal price is equal to $\underline{v}$ if $J(x) \geq 0$ for each $x \in[\underline{v}, \bar{v}] . J(x)$ is often called the "virtual valuation" of type $x$ (Myerson, 1981) and represents the marginal contribution to M's profit made by the sale of the object to a buyer with valuation $x$, taking into account a negative effect on the payment the seller obtains from each type with valuation greater than $x$.

In our two-object setting, the virtual valuation for object $i$ of a type $\left(v_{1}, v_{2}\right)$ is $J_{i}\left(v_{i}\right) \equiv$ $v_{i}-\frac{1-F_{i}\left(v_{i}\right)}{f_{i}\left(v_{i}\right)}$ for $v_{i} \in\left[\underline{v}_{i}, \bar{v}_{i}\right]$ and $i=1,2$. Let $J_{i}^{m} \equiv \min _{x \in\left[\underline{v}_{i}, \bar{v}_{i}\right]} J_{i}(x)$. Hence, $J_{i}^{m} \geq 0$ is equivalent to $J_{i}\left(v_{i}\right) \geq 0$ for any $v_{i} \in\left[\underline{v}_{i}, \bar{v}_{i}\right]$. The next proposition establishes that the optimal pricing schedule for $\mathrm{M}$ consists in pure bundling when the virtual valuation for each object is non-negative for all types.

Proposition 1 Suppose that $v_{1}$ and $v_{2}$ are independently distributed. If $J_{1}^{m} \geq 0$ and $J_{2}^{m} \geq 0$, then for any given mixed bundling schedule, there is a pure bundling schedule satisfying $P=\min \left\{p_{1}+\underline{v}_{2}, p_{2}+\underline{v}_{1}\right\}$ which gives $M$ a higher profit. Therefore, M's profit is maximized by a pure bundling schedule.

Let $P^{*}$ denote the optimal pure bundling price, i.e. the solution to the problem of $\max _{P} P \operatorname{Pr}\left\{v_{1}+v_{2} \geq P\right\}$. Proposition 1 establishes that if $J_{1}^{m} \geq 0$ and $J_{2}^{m} \geq 0$, then $P^{*}$, jointly with $p_{1}=\min \left\{\bar{v}_{1}, P^{*}-\underline{v}_{2}\right\}$ and $p_{2}=\min \left\{\bar{v}_{2}, P^{*}-\underline{v}_{1}\right\}$, is the optimal pricing schedule since each mixed bundling schedule is suboptimal. ${ }^{11}$

In order to illustrate the main ideas of the proof of Proposition 1, we consider a mixed bundling schedule $\left(p_{1}, p_{2}, P\right)$ satisfying $p_{1}<\bar{v}_{1}, p_{2}<\bar{v}_{2}, p_{2}+\underline{v}_{1}<p_{1}+\underline{v}_{2}<P$ where we assume $p_{2}+\underline{v}_{1}<p_{1}+\underline{v}_{2}$ only to fix the ideas. With the help of Figure 1(a)-(b), we show that a small reduction in the price of the bundle from $P$ to $P^{\prime}=P-\varepsilon$ (with $\varepsilon>0$ and small) is profitable. Figure 1 (a) represents the sets $S_{1}, S_{2}, S_{12}$ given the initial mixed bundling schedule. In Figure 1(b), we consider the reduction in the price of the bundle and partition $V$ into three subsets $X, Y, Z$ such that $X \equiv\left\{\left(v_{1}, v_{2}\right) \in V: v_{2} \geq p_{2}\right\}$,

\footnotetext{
${ }^{11}$ We specify $p_{1}=\min \left\{\bar{v}_{1}, P^{*}-\underline{v}_{2}\right\}$ and $p_{2}=\min \left\{\bar{v}_{2}, P^{*}-\underline{v}_{1}\right\}$ because of the restriction on $\left(p_{1}, p_{2}\right)$ that we previously imposed without loss of generality, i.e., $p_{1} \leq \min \left\{\bar{v}_{1}, P-\underline{v}_{2}\right\}$ and $p_{2} \leq \min \left\{\bar{v}_{2}, P-\underline{v}_{1}\right\}$. More generally, $P^{*}$ together with $p_{1} \geq \min \left\{\bar{v}_{1}, P^{*}-\underline{v}_{2}\right\}$ and $p_{2} \geq \min \left\{\bar{v}_{2}, P^{*}-\underline{v}_{1}\right\}$ is optimal.
} 
$Y \equiv\left\{\left(v_{1}, v_{2}\right) \in V: v_{2} \in\left[P-p_{1}, p_{2}\right)\right\}, Z \equiv\left\{\left(v_{1}, v_{2}\right) \in V: v_{2}<P-p_{1}\right\}$. We now prove that the reduction in the price of the bundle is profitable in each of the three regions $X, Y, Z$.

[Please put Figure 1 about here]

Caption for Figure 1: Illustration of the proof of Proposition 1

First, regarding the region $Z$, it is straightforward to see that the reduction in the price of the bundle is profitable because it induces some types in $Z$ to buy the bundle rather than buying nothing, or buying only object 1 . Second, regarding the region $X$, notice that every type in this set buys at least object 2 under $\left(p_{1}, p_{2}, P\right)$. For any type buying object 2 , the implicit price of object 1 is $P-p_{2}$; therefore a type in $X$ buys also object 1 (i.e., buys the bundle) if and only if $v_{1} \geq P-p_{2}$. Hence, for the types in $X$, the reduction in the price of the bundle has the effect of reducing the (implicit) price of object 1 and $J_{1}\left(P-p_{2}\right) \geq J_{1}^{m} \geq 0$ implies that the reduction increases M's profit from the types in $X$. Last, regarding $Y$, for each given $v_{2} \in\left[P-p_{1}, p_{2}\right)$, let $Y\left(v_{2}\right)=\left\{\left(v_{1}, v_{2}\right)\right.$ such that $\left.v_{1} \in\left[\underline{v}_{1}, \bar{v}_{1}\right]\right\}$ be the horizontal segment in $V$ such that the valuation for object 2 is equal to $v_{2}$; thus $Y=\cup_{v_{2} \in\left[P-p_{1}, p_{2}\right)} Y\left(v_{2}\right)$. Each type in $Y\left(v_{2}\right)$ buys the bundle if $v_{1} \geq P-v_{2}$, buys nothing if $v_{1}<P-v_{2}$. Reducing the price of the bundle from $P$ to $P^{\prime}$ has an effect on the types in $Y\left(v_{2}\right)$ similar to the effect on the types in region $X$, but the profit increase from the types in $Y\left(v_{2}\right)$ who buy the bundle under $\left(p_{1}, p_{2}, P^{\prime}\right)$ but buy nothing under $\left(p_{1}, p_{2}, P\right)$ is $P^{\prime}$, which is larger than $P^{\prime}-p_{2}$, the profit increase from the types in $X$ who buy the bundle under $\left(p_{1}, p_{2}, P^{\prime}\right)$ but buy only object 2 under $\left(p_{1}, p_{2}, P\right)$.

Formally, we find that

$$
\begin{aligned}
\frac{\partial \pi}{\partial P}= & {\left[1-F_{2}\left(p_{2}\right)\right]\left[1-F_{1}\left(P-p_{2}\right)-\left(P-p_{2}\right) f_{1}\left(P-p_{2}\right)\right] } \\
& +\int_{P-p_{1}}^{p_{2}}\left[1-F_{1}\left(P-v_{2}\right)-P f_{1}\left(P-v_{2}\right)\right] f_{2}\left(v_{2}\right) d v_{2}-\left(P-p_{1}\right)\left[1-F_{1}\left(p_{1}\right)\right] f_{2}\left(P-p_{1}\right),
\end{aligned}
$$

where each of the first, the second, the third terms refers, respectively, to region $X, Y, Z$. Notice that $J_{1}^{m} \geq 0$ implies $1-F_{1}\left(P-p_{2}\right)-\left(P-p_{2}\right) f_{1}\left(P-p_{2}\right) \leq 0$ and $\int_{P-p_{1}}^{p_{2}}\left[1-F_{1}(P-\right.$ $\left.\left.v_{2}\right)-P f_{1}\left(P-v_{2}\right)\right] f_{2}\left(v_{2}\right) d v_{2} \leq 0$. Therefore $\frac{\partial \pi}{\partial P}<0$ since the third term term is strictly negative.

If we consider a mixed bundling schedule such that $p_{1}<\bar{v}_{1}, p_{2}<\bar{v}_{2}$, and $p_{2}+\underline{v}_{1}<P=$ $p_{1}+\underline{v}_{2}$ instead of $p_{2}+\underline{v}_{1}<p_{1}+\underline{v}_{2}<P$, we find again that a reduction in the price of the bundle is profitable because (i) region $Z$ is empty in this case; (ii) in regions $X$ and $Y$ the 
previous arguments still apply (in the proof of Proposition 1 we take care of an extreme case in which $\left.\frac{\partial \pi}{\partial P}=0\right) \cdot{ }^{12}$

We note that the optimal pure bundling price $P^{*}$ is larger than $\underline{v}_{1}+\underline{v}_{2}$, as is shown by Armstrong (1996), even when $J_{1}^{m} \geq 0$ and $J_{2}^{m} \geq 0$ hold. In fact, if we let $G$ and $g$ denote the c.d.f. and the density of $v_{1}+v_{2}$, then $g\left(\underline{v}_{1}+\underline{v}_{2}\right)=0$. Therefore, under pure bundling, the virtual valuation for the bundle of a type with $v_{1}+v_{2}$ close to $\underline{v}_{1}+\underline{v}_{2}$ is negative, and $P^{*}>\underline{v}_{1}+\underline{v}_{2}$. This implies that there always exists a positive measure of types who buy nothing in the optimal pure bundling schedule.

Remarks on non-negative virtual valuations Given the assumptions in Proposition 1 , here we provide two remarks about distributions such that. $J_{1}^{m} \geq 0$.

First, suppose that (i) $G_{1}$ is the c.d.f. of a random variable with support $\left[\underline{\nu}_{1}, \bar{\nu}_{1}\right]$ and a strictly positive and continuous density $g_{1}$; (ii) $v_{1}$ has support $\left[\underline{v}_{1}, \bar{v}_{1}\right]$ such that $\underline{v}_{1}=\underline{\nu}_{1}+\omega$, $\bar{v}_{1}=\bar{\nu}_{1}+\omega$ for some $\omega>0$, and has a c.d.f. $F_{1}$ which is a $\omega$-rightward shift of $G_{1}$. Then $J_{1}^{m} \geq 0$ if $\omega$ is larger than $-\min _{x \in\left[\underline{\nu}_{1}, \bar{\nu}_{1}\right]}\left(x-\frac{1-G_{1}(x)}{g_{1}(x)}\right)$. In words, a sufficiently large rightward shift makes positive the virtual valuation for object 1 . An intuition for this result is immediate. First notice that $x-\frac{1-G_{1}(x)}{g_{1}(x)} \geq 0$ is equivalent to $x g_{1}(x) \geq 1-G_{1}(x)$, which compares the gain from selling the object to a type with valuation $x$, which is $x g_{1}(x)$, with the loss from types with higher valuation, which is $1-G_{1}(x)$. Then we observe that after an $\omega$-rightward shift in the distribution, the virtual valuation of a type $\omega+x$ (with $x \in\left[\underline{\nu}_{1}, \bar{\nu}_{1}\right]$ ) is non-negative if and only if $(\omega+x) f_{1}(x+\omega) \geq 1-F_{1}(x+\omega)$, that is if and only if $(\omega+x) g_{1}(x) \geq 1-G_{1}(x)$, which is definitely satisfied for a large $\omega$. Essentially, an $\omega-$ rightward shift increases the gain from selling the object to any type without affecting the loss from types with higher valuation.

Second, in a certain sense it is simpler to satisfy the inequality $J_{1}^{m} \geq 0$ if the density is decreasing than if it is increasing. Precisely, suppose that $f_{1}$ is increasing, and that $J_{1}^{m} \geq 0$, which is equivalent to $\underline{v}_{1} f_{1}\left(\underline{v}_{1}\right) \geq 1$. Then consider the density $g_{1}$ with support $\left[\underline{v}_{1}, \bar{v}_{1}\right]$ and

\footnotetext{
${ }^{12}$ Notice that we have used only the assumption $J_{1}^{m} \geq 0$, and not $J_{2}^{m} \geq 0$, because the initial mixed bundling schedule is such that $p_{2}+\underline{v}_{1} \leq p_{1}+\underline{v}_{2}$. Conversely, if $p_{1}+\underline{v}_{2}<p_{2}+\underline{v}_{1}$, then $J_{1}^{m} \geq 0$ (without $J_{2}^{m} \geq 0$ ) implies that $P$ can be profitably reduced to $p_{2}+\underline{v}_{1}$ (hence $\mu_{2}=0$ ), but not that $P$ should be reduced to $p_{1}+\underline{v}_{2}$. For instance, if $\left(v_{1}, v_{2}\right)$ is uniformly distributed over $[6,7] \times[0,10]$, then $J_{1}^{m}=5>0>-10=J_{2}^{m}$ and the maximal profit under pure bundling is 6.806 (with $P=8.25$ ), but the schedule $p_{1}=6, p_{2}=5$, $P=11$ implies $\mu_{1}=\frac{1}{2}, \mu_{2}=0, \mu_{12}=\frac{1}{2}$ and therefore the profit is $8.5(>6.806)$.
} 
such that $g_{1}(x)=f_{1}\left(\underline{v}_{1}+\bar{v}_{1}-x\right)$ for each $x \in\left[\underline{v}_{1}, \bar{v}_{1}\right]$; therefore $g_{1}$ is decreasing and its graph is the mirror image of the graph of $f_{1}$ with respect to the vertical line $x=\frac{1}{2}\left(\underline{v}_{1}+\bar{v}_{1}\right)$. Letting $G_{1}(x)=\int_{\underline{v}_{1}}^{x} g_{1}(z) d z$, we find that $x-\frac{1-G_{1}(x)}{g_{1}(x)} \geq 0$ is equivalent to $x g_{1}(x) \geq 1-G_{1}(x)$, and $x g_{1}(x) \geq \underline{v}_{1} g_{1}\left(\bar{v}_{1}\right)=\underline{v}_{1} f_{1}\left(\underline{v}_{1}\right) \geq 1$. Thus $J_{1}^{m} \geq 0$ with an increasing $f_{1}$ implies that $\min _{x \in\left[\underline{\nu}_{1}, \bar{\nu}_{1}\right]}\left(x-\frac{1-G_{1}(x)}{g_{1}(x)}\right) \geq 0$, for a distribution with a density which is the mirror image of $f_{1}$ (and thus decreasing). This occurs since $J_{1}^{m} \geq 0$ is equivalent to $x f_{1}(x) \geq 1-F_{1}(x)$ for each $x \in\left[\underline{v}_{1}, \bar{v}_{1}\right]$, which holds if and only if the gain $\underline{v}_{1} f_{1}\left(\underline{v}_{1}\right)$ from selling to type $\underline{v}_{1}$ is larger than 1 . The gain from selling to low types (like $\underline{v}_{1}$ ) is larger for density $g_{1}$ than for $f_{1}$ : in fact with density $g_{1}$, for any $x$ in $\left[\underline{v}_{1}, \bar{v}_{1}\right]$ the gain from selling to type $x$ cannot be smaller than $\underline{v}_{1} f_{1}\left(\underline{v}_{1}\right)$, which we know to be at least one. Furthermore, the information rent given to the types with valuation higher than $x$ is greater with $F_{1}$ than with $G_{1}: 1-F_{1}(x)>1-G_{1}(x)$.

Extensions If $\mathrm{M}$ has valuations (or production costs) $c_{1}, c_{2}$ for the two objects, then the result in Proposition 1 holds if $J_{1}^{m} \geq c_{1}$ and $J_{2}^{m} \geq c_{2}$.

If $v_{1}, v_{2}$ are not independently distributed, then Proposition 1 extends in a natural way. Precisely, letting $f_{i \mid j}$ and $F_{i \mid j}$ denote the conditional density and the conditional c.d.f. of $v_{i}$ given $v_{j}$ (for $i, j=1,2, i \neq j$ ), mixed bundling is suboptimal if $v_{i}-\frac{1-F_{i \mid j}\left(v_{i} \mid v_{j}\right)}{f_{i \mid j}\left(v_{i} \mid v_{j}\right)} \geq 0$ for any $\left(v_{i}, v_{j}\right) \in V$, for $i, j=1,2, i \neq j$.

Relationship with McAfee, McMillan and Whinston (1989) McAfee et al. (1989) consider the same model we have studied (allowing for correlated valuations). Their main result is that any independent pricing schedule (i.e., such that $P=p_{1}+p_{2}$ ) is suboptimal under a suitable restriction on the distribution of $v_{1}, v_{2}$, which is always satisfied by independent distributions. But they do not study conditions under which pure bundling generates the highest profit. Precisely, let $p_{1}^{*}, p_{2}^{*}$ denote the optimal prices under independent pricing. Then, they show that the schedule $\left(p_{1}^{*}, p_{2}^{*}, p_{1}^{*}+p_{2}^{*}-\varepsilon\right)$ is superior to $\left(p_{1}^{*}, p_{2}^{*}, p_{1}^{*}+p_{2}^{*}\right)$. Hence, any independent pricing schedule is inferior to a suitable mixed bundling schedule. An implicit assumption in their analysis is that $p_{1}^{*}$ and $p_{2}^{*}$ are such that, in our notation, $p_{1}^{*}>\underline{v}_{1}$ and $p_{2}^{*}>\underline{v}_{2}$. Conversely, our assumptions $J_{1}^{m} \geq 0$ and $J_{2}^{m} \geq 0$ imply $p_{1}^{*}=\underline{v}_{1}$ and $p_{2}^{*}=\underline{v}_{2}$, and hence, in our setting, reducing $P$ below $p_{1}^{*}+p_{2}^{*}$ definitely reduces M's profit. Rather, Proposition 1 proves that $\mathrm{M}$ should choose the optimal pure bundling price and combine it with $p_{1}, p_{2}$ sufficiently high to make $\mu_{1}=\mu_{2}=0$. 
The case of i.i.d. valuations Here we consider the case in which $v_{1}$ and $v_{2}$ are i.i.d., each with support $[\underline{v}, \bar{v}]$, c.d.f. $F$, and density $f$; hence $\mathrm{M}$ can focus on $\left(p_{1}, p_{2}, P\right)$ satisfying $p_{1}=p_{2} \equiv p$. We define $J(x) \equiv x-\frac{1-F(x)}{f(x)}$ and $J^{m} \equiv \min _{x \in[\underline{v}, \bar{v}]} J(x)$.

Proposition 2 Suppose that $v_{1}$ and $v_{2}$ are independently distributed with $\underline{v}_{1}=\underline{v}_{2} \equiv \underline{v}$, $\bar{v}_{1}=\bar{v}_{2} \equiv \bar{v}$, and $F_{1}=F_{2} \equiv F$. If $\underline{v}+J^{m} \geq 0$, then for any given mixed bundling schedule, there is a pure bundling schedule satisfying $P=p+\underline{v}$ which gives $M$ a higher profit. Therefore, M's profit is maximized by a pure bundling schedule.

Proposition 2 strengthens the result in Proposition 1 for the specific case of i.i.d. valuations, as it establishes that mixed bundling is suboptimal even though $J(x)<0$ for some $x$, provided that $\underline{v}+J^{m} \geq 0$. The result follows because $F_{1}=F_{2}$ allows to combine the first and the third term of $\frac{\partial \pi}{\partial P}$ in $(1)$ to prove that $\frac{\partial \pi}{\partial P}$ is negative even though sometimes the first term is positive.

From Proposition 2 and the remarks on non-negative virtual valuations, we obtain:

Corollary 1 Consider an increasing density $f$ with support $[\underline{v}, \bar{v}]$ and such that $2 \underline{v} f(\underline{v}) \geq 1$

(i) Suppose that $v_{1}$ and $v_{2}$ are independently distributed with the identical support $[\underline{v}, \bar{v}]$ and common density $f$. Then M's profit is maximized by a pure bundling schedule.

(ii) Suppose that $v_{1}$ and $v_{2}$ are independently distributed with the identical support $[\underline{v}, \bar{v}]$ and common decreasing density $g$, of which the graph is the mirror image of the graph of $f$ with respect to the vertical line $x=\frac{1}{2}(\underline{v}+\bar{v})$. Then, M's profit is maximized by a pure bundling schedule.

In the case of uniform distribution with support $[\underline{v}, \bar{v}]$, pure bundling is optimal if $\underline{v} \geq \frac{1}{3} \bar{v}$.

\section{General mechanisms}

In Section 3 we have focused on the class of selling mechanisms such that $M$ chooses a price schedule, which specifies a price for each object and a price for the bundle. However, if we consider the set of all incentive compatible and individually rational mechanisms, which we denote by $\mathcal{M}$, then $\mathcal{M}$ includes many other mechanisms, and in particular stochastic mechanisms specifying that certain types of buyer receive an object with a probability in $(0,1)$. In this section we provide sufficient conditions for pure bundling to be the optimal 
selling mechanism among all mechanisms in $\mathcal{M}$. We restrict attention to the case of i.i.d. valuations with c.d.f. $F$, density $f$ for each valuation, and $V=[\underline{v}, \bar{v}] \times[\underline{v}, \bar{v}]$.

From the Revelation Principle, we can describe a generic mechanism in $\mathcal{M}$ in terms of two functions, $q=\left(q_{1}, q_{2}\right): V \rightarrow[0,1] \times[0,1]$ and $T: V \rightarrow \mathbb{R}$, such that a buyer reporting valuations $v^{\prime}=\left(v_{1}^{\prime}, v_{2}^{\prime}\right)$ receives object 1 with probability $q_{1}\left(v^{\prime}\right)$, receives object 2 with probability $q_{2}\left(v^{\prime}\right)$, and pays $T\left(v^{\prime}\right)$. The objective of $\mathrm{M}$ is to choose $q$ and $T$ in order to maximize the expectation of $T(v)$ subject to participation and incentive constraints:

$$
v_{1} q_{1}(v)+v_{2} q_{2}(v)-T(v) \geq \max \left\{0, v_{1} q_{1}\left(v^{\prime}\right)+v_{2} q_{2}\left(v^{\prime}\right)-T\left(v^{\prime}\right)\right\} \text { for each } v \text { and } v^{\prime} \text { in } V \text {. }
$$

Although this is typically a complicated problem, the results in Pavlov (2011) give some insights on the optimal mechanism. First, since $v_{1}, v_{2}$ are identically distributed, we can focus on determining the optimal $q_{1}, q_{2}, T$ for $\left(v_{1}, v_{2}\right)$ such that $v_{2} \geq v_{1}$; symmetric results are obtained for $v_{2}<v_{1}$. Second, Pavlov (2011) shows that under the condition

$$
3+v_{1} \frac{f^{\prime}\left(v_{1}\right)}{f\left(v_{1}\right)}+v_{2} \frac{f^{\prime}\left(v_{2}\right)}{f\left(v_{2}\right)} \geq 0 \text { for each }\left(v_{1}, v_{2}\right) \in V,
$$

it is optimal for $\mathrm{M}$ to restrict attention to mechanisms in which the buyer either gets no object, or gets her most preferred object for sure and her less preferred object with some probability. ${ }^{13}$

Formally, given $v_{2} \geq v_{1}$, either $q_{1}\left(v_{1}, v_{2}\right)=q_{2}\left(v_{1}, v_{2}\right)=0$, or $q_{2}\left(v_{1}, v_{2}\right)=1$ and $q_{1}\left(v_{1}, v_{2}\right) \in$ $[0,1]$. Furthermore, there is no loss for $\mathrm{M}$ in performing the screening only on the valuation $v_{1}$ for object 1 , and in optimizing over mechanisms characterized by two functions, $q_{1}:[\underline{v}, \bar{v}] \rightarrow$ $[0,1]$ and $t:[\underline{v}, \bar{v}] \rightarrow \mathbb{R}$, and in which the buyer chooses a message $v_{1}^{\prime}$ in $[\underline{v}, \bar{v}] \cup\{\varnothing\} .{ }^{14}$ If $v_{1}^{\prime}=\varnothing$, then the buyer does not participate: she receives no object and pays zero. If conversely $v_{1}^{\prime} \in[\underline{v}, \bar{v}]$, then the buyer receives object 2 with probability 1 , receives object 1 with probability $q_{1}\left(v_{1}^{\prime}\right)$, and pays $t\left(v_{1}^{\prime}\right)$. Let $u\left(v_{1}\right) \equiv v_{1} q_{1}\left(v_{1}\right)-t\left(v_{1}\right)$. Then, the payoff of a type $v=\left(v_{1}, v_{2}\right)$ reporting $v_{1}^{\prime}=v_{1}$ is $u\left(v_{1}\right)+v_{2}$; therefore type $v$ participates if and only if $u\left(v_{1}\right)+v_{2} \geq 0$.

\footnotetext{
${ }^{13}$ Condition (2) is a sort of hazard rate condition that appears in some literature on multidimentional mechanism design: see McAfee and McMillan (1988) and Manelli and Vincent (2006).

${ }^{14}$ Here, we are somewhat abusing notation by using again $q_{1}$ to denote a function defined on $[\underline{v}, \bar{v}]$, whereas $q_{1}$ which was introduced previously is defined on $V$. However, since only $q_{1}:[\underline{v}, \bar{v}] \rightarrow[0,1]$ is used from now on, there is no concern about ambiguity.
} 
The profit of $\mathrm{M}$ from type $v$ is $t\left(v_{1}\right)=v_{1} q_{1}\left(v_{1}\right)-u\left(v_{1}\right)$ if $u\left(v_{1}\right)+v_{2} \geq 0$, but is 0 otherwise. The expected profit is

$$
\int_{\underline{v}}^{\bar{v}}\left[v_{1} q_{1}\left(v_{1}\right)-u\left(v_{1}\right)\right] f\left(v_{1}\right)\left[1-F\left(\max \left\{v_{1},-u\left(v_{1}\right)\right\}\right)\right] d v_{1},
$$

in which $1-F\left(\max \left\{v_{1},-u\left(v_{1}\right)\right\}\right)$ takes into account that we are considering types satisfying $v_{2} \geq v_{1}$ and that only types satisfying $v_{2} \geq-u\left(v_{1}\right)$ participate. Standard techniques show that the incentive constraints in this problem are satisfied if and only if $q_{1}$ is weakly increasing and $u\left(v_{1}\right)=u(\underline{v})+\int_{\underline{v}}^{v_{1}} q_{1}(x) d x$ for each $v_{1} \in[\underline{v}, \bar{v}]$. Therefore, $u$ is increasing, and if $u(\underline{v}) \geq-\underline{v}$, then $v_{1} \geq-u\left(v_{1}\right)$ for each $v_{1} \in[\underline{v}, \bar{v}]$, and hence each type participates since we are considering $v_{1}, v_{2}$ such that $v_{2} \geq v_{1}$.

The design problem is then reduced to maximizing (3) with respect to $u(\underline{v})$ (within the set $\mathbb{R}$ ) and with respect to $q_{1}$ (within the set of weakly increasing functions with domain $[\underline{v}, \bar{v}]$ and codomain $[0,1])$. This is a one-dimensional screening problem in which the screening variable is the probability that the buyer receives her less preferred object, as a function of her reported valuation for that object. A non-standard feature is that for each type of buyer with valuation $v_{1}$ for object 1 , her participation is determined by the sign of $u\left(v_{1}\right)+v_{2}$, in which $v_{2}$ is private information of the buyer.

In this setting, pure bundling is obtained if $q_{1}\left(v_{1}\right)=1$ for each $v_{1} \in[\underline{v}, \bar{v}]$, since then each type either receives both objects (if she participates), or receives no object (if she does not participate). In this case the bundle price is $\underline{v}-u(\underline{v})$, selected by M through the choice of $u(\underline{v})$.

Proposition 3 Suppose that $v_{1}, v_{2}$ are i.i.d., each with support $[\underline{v}, \bar{v}]$ and a density $f$ which satisfies (2), and that there exists $\beta>1$ satisfying the two following conditions:

$$
\begin{gathered}
x f(x) \geq \beta \quad \text { for each } x \in[\underline{v}, \bar{v}] ; \\
F\left(\frac{1}{2} \underline{v}+\frac{1}{2} \bar{v}\right) \leq \beta-1 .
\end{gathered}
$$

Then the optimal pure bundling price schedule is the optimal mechanism among all mechanisms in $\mathcal{M}$.

Since $J^{m} \geq 0$ is equivalent to $x f(x) \geq 1-F(x)$ for each $x \in[\underline{v}, \bar{v}]$, it follows that condition (4) can be interpreted as a strengthening of $J^{m} \geq 0$, which is more restrictive the 
greater is $\beta$. Conversely, (5) is less restrictive the greater is $\beta$, and it puts an upper bound on the probability mass in the left half interval of $[\underline{v}, \bar{v}]$. Inequality (5) is used in the proof of Proposition 3 to show that, although $u(\underline{v})$ is smaller than $-\underline{v}$, it is not too smaller than $-\underline{v}$, and this helps to make pure bundling optimal.

It is immediate to see that if $f$ is increasing and $\beta=\frac{3}{2}$, then (i) (2) is satisfied; (ii) (4) is equivalent to $\underline{v} f(\underline{v}) \geq \frac{3}{2}$; (iii) (5) holds since $f$ increasing implies $F$ convex, thus $F\left(\frac{1}{2} \underline{v}+\frac{1}{2} \bar{v}\right) \leq \frac{1}{2} F(\underline{v})+\frac{1}{2} F(\bar{v})=\frac{1}{2}$. Hence, the following corollary holds.

Corollary 2 Suppose that $v_{1}, v_{2}$ are i.i.d., each with support $[\underline{v}, \bar{v}]$ and an increasing density $f$ such that $\underline{v} f(\underline{v}) \geq \frac{3}{2}$. Then the optimal pure bundling price schedule is the optimal mechanism among all mechanisms in $\mathcal{M}$.

Notice that if (4) is satisfied for $\beta \geq 2$, then (5) holds even though $f$ is not increasing, but then (2) is not necessarily satisfied. ${ }^{15}$

Sufficient conditions for pure bundling to be the optimal mechanisms can be found also in Giannakopoulos (2014) and in Hart and Nisan (2014). Precisely, in the first paper valuations are i.i.d. with exponential density; ${ }^{16}$ in the second paper, $v_{1}, v_{2}$ are i.i.d. with a density $f$ such that $x^{3 / 2} f(x)$ is decreasing in $[\underline{v}, \bar{v}]$. Neither of these conditions implies the assumptions of Proposition 3, nor vice versa.

\section{Concluding remarks}

We have given sufficient conditions for pure bundling to be the optimal mechanism, both for the case in which the seller's available instruments are restricted to price schedules, and for the case in which he can choose any incentive compatible and individually rational mechanism. It is interesting that unrelated conditions like those in Proposition 3, and those in Giannakopoulos (2014) and Hart and Nisan (2014) all imply that pure bundling is optimal. An interesting question for future research is to know whether they are special cases of more general conditions under which pure bundling is optimal. Furthermore, little is known about the optimal mechanism to sell two (or more) objects when there are two (or more) buyers

\footnotetext{
${ }^{15}$ The remark in Section 3 about large rightward shifts applies to satisfy (4) with $\beta \geq 2$, but if the density's derivative is negative at some point, then (2) is violated for sufficiently large rightward shifts.

${ }^{16}$ The result by Giannakopoulos (2014) holds also for the case of more than two objects.
} 
which compete to buy the objects. Therefore it would be interesting to study whether the progress made for the setting with a single buyer helps to improve our understanding of the setting with multiple buyers. For instance, are there cases in which the optimal mechanism consists in auctioning the bundle of all the objects on sale?

\section{References}

[1] Armstrong, M. (1996). "Multiproduct Nonlinear Pricing", Econometrica 64, pp. 51-76.

[2] Giannakopoulos, Y. (2014). "Bounding Optimal Revenue in Multiple-Items Auctions", arXiv 1404.2832 .

[3] Hart, S., Nisan, N. (2014). "How Good Are Simple Mechanisms for Selling Multiple Goods?", The Hebrew University of Jerusalem, Center for Rationality, DP-666.

[4] Hart, S., Reny, P.J. (2013) "Maximal Revenue with Multiple Goods: Nonmonotonicity and Other Observations", The Hebrew University of Jerusalem, Center for Rationality, DP-630.

[5] Li, X., Yao, A.C.-C. (2013), "On Revenue Maximization for Selling Multiple Independently Distributed Items," Proceedings of the National Academy of Sciences 110, pp. $11232-11237$.

[6] McAfee, R.P, McMillan, J. (1988). "Multidimensional Incentive Compatibility and Mechanism Design," Journal of Economic Theory 46, pp. 335-354.

[7] McAfee, R.P, McMillan, J., Whinston, M. (1989). "Multiproduct Monopoly, Commodity Bundling, and Correlation of Values," Quarterly Journal of Economics 104, pp. 371-384.

[8] Manelli, A.M., Vincent, D. R. (2006). "Bundling as an Optimal Selling Mechanism for a Multiple-Good Monopolist," Journal of Economic Theory 127, pp. 1-35.

[9] Manelli, A.M., Vincent, D. R. (2007). "Multi-Dimensional Mechanism Design: Revenue Maximization and the Multiple Good Monopoly" Journal of Economic Theory 137, pp.153-185. 
[10] Manelli, A.M., Vincent, D. R. (2012). "Multidimensional mechanism design: Revenue maximization and the multiple-good monopoly. A corrigendum" Journal of Economic Theory 147, pp. 2492-2493.

[11] Myerson, Roger (1981). "Optimal Auction Design," Mathematics of Operations Research, 6(1), 58-73.

[12] Pavlov, Gregory (2011). "Optimal Mechanism for Selling Two Goods," The B.E. Journal of Theoretical Economics, 11 (Advances), Article 3.

[13] Riley, John and Richard Zeckhauser (1983). "Optimal selling strategies: when to haggle, when to hold firm", Quarterly Journal of Economics 98, pp. 267-289. 


\section{Appendix}

\subsection{Proof of Proposition 1}

We fix an arbitrary mixed bundling schedule $\left(p_{1}, p_{2}, P\right)$ and prove that if $J_{1}^{m} \geq 0, J_{2}^{m} \geq$ 0 , then it is profitable for $\mathrm{M}$ to reduce the price of the bundle to $\min \left\{p_{1}+\underline{v}_{2}, p_{2}+\underline{v}_{1}\right\}$, which means that $\mathrm{M}$ plays a pure bundling schedule; therefore no mixed bundling schedule maximizes $\pi \cdot{ }^{17}$ In order to fix the ideas, we consider $p_{1}, p_{2}$ such that $p_{2}+\underline{v}_{1} \leq p_{1}+\underline{v}_{2} \cdot{ }^{18}$ Hence, $\left(p_{1}, p_{2}, P\right)$ is a pure bundling schedule if $p_{2}+\underline{v}_{1}=P$, which implies that $p_{2}+\underline{v}_{1}<P$ needs to hold if $\left(p_{1}, p_{2}, P\right)$ is a mixed bundling schedule. Step 1 in this proof proves that $\frac{\partial \pi}{\partial P}<0$ if $p_{1}+\underline{v}_{2}<P$. Step 2 considers the case of $p_{2}+\underline{v}_{1}<P=p_{1}+\underline{v}_{2}{ }^{19}$

Step 1 If $J_{1}^{m} \geq 0$ and $\left(p_{1}, p_{2}, P\right)$ is a mixed bundling schedule such that $p_{1}+\underline{v}_{2}<P$, then $\frac{\partial \pi}{\partial P}<0$.

Proof of Step 1. Since $\frac{\partial \pi}{\partial P}=\frac{\partial \mu_{1}}{\partial P} p_{1}+\frac{\partial \mu_{2}}{\partial P} p_{2}+\frac{\partial \mu_{12}}{\partial P} P+\mu_{12}$ and

$$
\begin{aligned}
& \frac{\partial \mu_{1}}{\partial P}=\left[1-F_{1}\left(p_{1}\right)\right] f_{2}\left(P-p_{1}\right), \frac{\partial \mu_{2}}{\partial P}=\left[1-F_{2}\left(p_{2}\right)\right] f_{1}\left(P-p_{2}\right), \\
& \frac{\partial \mu_{12}}{\partial P}=-\left[1-F_{1}\left(p_{1}\right)\right] f_{2}\left(P-p_{1}\right)-\left[1-F_{2}\left(p_{2}\right)\right] f_{1}\left(P-p_{2}\right)-\int_{P-p_{1}}^{p_{2}} f_{1}\left(P-v_{2}\right) f_{2}\left(v_{2}\right) d v_{2},
\end{aligned}
$$

after rearranging we obtain

$$
\begin{aligned}
\frac{\partial \pi}{\partial P}= & -\left(P-p_{1}\right)\left[1-F_{1}\left(p_{1}\right)\right] f_{2}\left(P-p_{1}\right)-\left(P-p_{2}\right)\left[1-F_{2}\left(p_{2}\right)\right] f_{1}\left(P-p_{2}\right)+ \\
& +\int_{P-p_{1}}^{p_{2}}\left[1-F_{1}\left(P-v_{2}\right)\right] f_{2}\left(v_{2}\right) d v_{2}+\left[1-F_{1}\left(P-p_{2}\right)\right]\left[1-F_{2}\left(p_{2}\right)\right] \\
& -P \int_{P-p_{1}}^{p_{2}} f_{1}\left(P-v_{2}\right) f_{2}\left(v_{2}\right) d v_{2} \\
= & {\left[1-F_{2}\left(p_{2}\right)\right]\left[1-F_{1}\left(P-p_{2}\right)-\left(P-p_{2}\right) f_{1}\left(P-p_{2}\right)\right]+} \\
& +\int_{P-p_{1}}^{p_{2}}\left[1-F_{1}\left(P-v_{2}\right)-P f_{1}\left(P-v_{2}\right)\right] f_{2}\left(v_{2}\right) d v_{2}-\left(P-p_{1}\right)\left[1-F_{1}\left(p_{1}\right)\right] f_{2}\left(P-p_{1}\right) .
\end{aligned}
$$

We know that $J_{1}(x) \geq 0$ for each $x \in\left[\underline{v}_{1}, \bar{v}_{1}\right]$, therefore $1-F_{1}\left(P-p_{2}\right)-\left(P-p_{2}\right) f_{1}\left(P-p_{2}\right) \leq 0$, and $\int_{P-p_{1}}^{p_{2}}\left[1-F_{1}\left(P-v_{2}\right)-P f_{1}\left(P-v_{2}\right)\right] f_{2}\left(v_{2}\right) d v_{2} \leq \int_{P-p_{1}}^{p_{2}}\left[1-F_{1}\left(P-v_{2}\right)-\left(P-v_{2}\right) f_{1}(P-\right.$

\footnotetext{
${ }^{17}$ Although in several cases we prove that $\frac{\partial \pi}{\partial P}<0$, in one case it is conceivable that $\frac{\partial \pi}{\partial P}=0$, so that reducing the price of the bundle has no effect on M's profit. However, in this case we still prove that the optimal pure bundling schedule is superior to the initial mixed bundling schedule.

${ }^{18}$ Given $p_{2}+\underline{v}_{1} \leq p_{1}+\underline{v}_{2}$, the inequality $J_{1}^{m} \geq 0$ suffices to prove the result. If $p_{2}+\underline{v}_{1}>p_{1}+\underline{v}_{2}$, then the inequality $J_{2}^{m} \geq 0$ suffices.

${ }^{19}$ We do not need to consider $P<p_{1}+\underline{v}_{2}$ since, as we explained in the main text, we assume without loss of generality $p_{1} \leq \min \left\{\bar{v}_{1}, P-\underline{v}_{2}\right\}$.
} 
$\left.\left.v_{2}\right)\right] f_{2}\left(v_{2}\right) d v_{2} \leq 0$. Hence, each term in $\frac{\partial \pi}{\partial P}$ is negative or zero and now we rule out the possibility of $\frac{\partial \pi}{\partial P}=0$. If $p_{1}<\bar{v}_{1}$, then $\frac{\partial \pi}{\partial P}<0$ because the third term in $\frac{\partial \pi}{\partial P}$ is negative. If $p_{1}=$ $\bar{v}_{1}$, then $p_{2}<\bar{v}_{2}$ in order for $\left(p_{1}, p_{2}, P\right)$ to be a mixed bundling schedule, and we distinguish the case of $P=p_{1}+p_{2}$ from the case of $P<p_{1}+p_{2}$. When $P=p_{1}+p_{2}$, the first term in $\frac{\partial \pi}{\partial P}$ is equal to $-\left[1-F_{2}\left(p_{2}\right)\right] \bar{v}_{1} f_{1}\left(\bar{v}_{1}\right)$, which is negative. When $P<p_{1}+p_{2}$, the second term in $\frac{\partial \pi}{\partial P}$ is equal to $-\int_{P-p_{1}}^{p_{2}} v_{2} f_{1}\left(P-v_{2}\right) f_{2}\left(v_{2}\right) d v_{2}+\int_{P-p_{1}}^{p_{2}}\left[1-F_{1}\left(P-v_{2}\right)-\left(P-v_{2}\right) f_{1}\left(P-v_{2}\right)\right] f_{2}\left(v_{2}\right) d v_{2}$, which is negative.

Step 2 If $J_{1}^{m} \geq 0$ and $\left(p_{1}, p_{2}, P\right)$ is a mixed bundling schedule such that $p_{2}+\underline{v}_{1}<$ $P=p_{1}+\underline{v}_{2}$, then there exists a pure bundling schedule which yields a higher profit than $\left(p_{1}, p_{2}, P\right)$.

Proof of Step 2. Given $P=p_{1}+\underline{v}_{2}$, we have $\mu_{1}=0, \mu_{2}=\left[1-F_{2}\left(p_{2}\right)\right] F_{1}\left(P-p_{2}\right)$, $\mu_{12}=\left[1-F_{1}\left(P-p_{2}\right)\right]\left[1-F_{2}\left(p_{2}\right)\right]+\int_{\underline{v}_{2}}^{p_{2}}\left[1-F_{1}\left(P-v_{2}\right)\right] f_{2}\left(v_{2}\right) d v_{2}$. Since $\frac{\partial \pi}{\partial P}=\frac{\partial \mu_{2}}{\partial P} p_{2}+\frac{\partial \mu_{12}}{\partial P} P+\mu_{12}$ and

$\frac{\partial \mu_{2}}{\partial P}=\left[1-F_{2}\left(p_{2}\right)\right] f_{1}\left(P-p_{2}\right), \quad \frac{\partial \mu_{12}}{\partial P}=-\left[1-F_{2}\left(p_{2}\right)\right] f_{1}\left(P-p_{2}\right)-\int_{\underline{v}_{2}}^{p_{2}} f_{1}\left(P-v_{2}\right) f_{2}\left(v_{2}\right) d v_{2}$,

after rearranging we obtain

$$
\begin{aligned}
\frac{\partial \pi}{\partial P}= & -\left(P-p_{2}\right)\left[1-F_{2}\left(p_{2}\right)\right] f_{1}\left(P-p_{2}\right)+\left[1-F_{1}\left(P-p_{2}\right)\right]\left[1-F_{2}\left(p_{2}\right)\right] \\
& +\int_{\underline{v}_{2}}^{p_{2}}\left[1-F_{1}\left(P-v_{2}\right)-P f_{1}\left(P-v_{2}\right)\right] f_{2}\left(v_{2}\right) d v_{2} \\
= & {\left[1-F_{2}\left(p_{2}\right)\right]\left[1-F_{1}\left(P-p_{2}\right)-\left(P-p_{2}\right) f_{1}\left(P-p_{2}\right)\right] } \\
& +\int_{\underline{v}_{2}}^{p_{2}}\left[1-F_{1}\left(P-v_{2}\right)-P f_{1}\left(P-v_{2}\right)\right] f_{2}\left(v_{2}\right) d v_{2}
\end{aligned}
$$

We can argue as in the proof of Step 1 to infer that each term in $\frac{\partial \pi}{\partial P}$ is negative or zero. Moreover, notice that if $p_{2}>\underline{v}_{2}$ then $\frac{\partial \pi}{\partial P}<0$ because the second term in $\frac{\partial \pi}{\partial P}$ is equal to $-\int_{\underline{v}_{2}}^{p_{2}} v_{2} f_{1}\left(P-v_{2}\right) f_{2}\left(v_{2}\right) d v_{2}+\int_{\underline{v}_{2}}^{p_{2}}\left[1-F_{1}\left(P-v_{2}\right)-\left(P-v_{2}\right) f_{1}\left(P-v_{2}\right)\right] f_{2}\left(v_{2}\right) d v_{2}$, which is negative. If conversely $p_{2}=\underline{v}_{2}$, then it is possible that $\frac{\partial \pi}{\partial P}=0$ and that decreasing the price of the bundle until $\underline{v}_{1}+\underline{v}_{2}$ has no effect on $\pi$. In such a case, the profit under the initial mixed bundling schedule coincides with $\underline{v}_{1}+\underline{v}_{2}$, but we know that the optimal pure bundling schedule yields a profit higher than $\underline{v}_{1}+\underline{v}_{2}$, and thus higher than the profit under the initial mixed bundling schedule. Therefore, even in this case we can find a pure bundling schedule which is superior to the initial mixed bundling schedule. 


\subsection{Proof of Proposition 2}

Using the symmetry in the distributions and $p_{1}=p_{2}=p$, we obtain $\mu_{1}=\mu_{2}=[1-$ $F(p)] F(P-p), \mu_{12}=\int_{P-p}^{p}\left[1-F\left(P-v_{2}\right)\right] f\left(v_{2}\right) d v_{2}+[1-F(P-p)][1-F(p)]$, and $\pi=$ $2 p \mu_{1}+P \mu_{12}$. Therefore

$\frac{\partial \pi}{\partial P}=[1-F(p)][1-F(P-p)-2(P-p) f(P-p)]+\int_{P-p}^{p}\left[1-F\left(P-v_{2}\right)-P f\left(P-v_{2}\right)\right] f\left(v_{2}\right) d v_{2}$.

In order for $(p, P)$ to be a mixed bundling schedule it is necessary that $p<\bar{v}$ and $P-p>\underline{v}$. Hence the first term in $\frac{\partial \pi}{\partial P}$ is negative since $1-F(p)>0$, and $1-F(P-p)-2(P-p) f(P-p)<$ 0 is equivalent to $P-p+J(P-p)>0$, which is satisfied since $P-p>\underline{v}, J(P-p) \geq J^{m}$, and $\underline{v}+J^{m} \geq 0$; (ii) $1-F\left(P-v_{2}\right)-P f\left(P-v_{2}\right) \leq 0$ is equivalent to $v_{2}+J\left(P-v_{2}\right) \geq 0$, which is satisfied since $\underline{v}+J^{m} \geq 0$.

\subsection{The case of $P>p_{1}+p_{2}$}

In the case of $P>p_{1}+p_{2}$, we assume that the seller can monitor the buyer's purchase such that when she buys both objects she must pay $P$. This assumption is not needed to be explicitly made when $P \leq p_{1}+p_{2}$ since the buyer prefers paying $P$ to paying $p_{1}+p_{2}$ when she buys both objects.

For price schedules such that $P>p_{1}+p_{2}$, we can argue as in Section 3 to find that a buyer of type $\left(v_{1}, v_{2}\right)$ buys only object 1 if and only if $v_{1} \geq p_{1}, v_{2}<P-p_{1}, v_{2}<p_{2}-p_{1}+v_{1}$; buys only object 2 if and only if $v_{2} \geq p_{2}, v_{1}<P-p_{2}, v_{2} \geq p_{2}-p_{1}+v_{1}$; buys the bundle if and only if $v_{1} \geq P-p_{2}, v_{2} \geq P-p_{1}$. Moreover, without loss of generality we can restrict to $\left(p_{1}, p_{2}, P\right)$ which satisfy $p_{1} \leq \min \left\{\bar{v}_{1}, P-\underline{v}_{2}\right\}, p_{2} \leq \min \left\{\bar{v}_{2}, P-\underline{v}_{1}\right\},{ }^{20}$ and $P-p_{1} \leq \bar{v}_{2}$, $P-p_{2} \leq \bar{v}_{1} \cdot{ }^{21}$

Hence

$$
\begin{aligned}
\mu_{1} & =\int_{p_{1}}^{P-p_{2}} f_{1}\left(v_{1}\right) F_{2}\left(p_{2}-p_{1}+v_{1}\right) d v_{1}+\left[1-F_{1}\left(P-p_{2}\right)\right] F_{2}\left(P-p_{1}\right), \\
\mu_{2} & =\int_{p_{1}}^{P-p_{2}} f_{1}\left(v_{1}\right)\left[1-F_{2}\left(p_{2}-p_{1}+v_{1}\right)\right] d v_{1}+F_{1}\left(p_{1}\right)\left[1-F_{2}\left(p_{2}\right)\right], \\
\mu_{12} & =\left[1-F_{1}\left(P-p_{2}\right)\right]\left[1-F_{2}\left(P-p_{1}\right)\right],
\end{aligned}
$$

\footnotetext{
${ }^{20}$ As in Section 3.

${ }^{21}$ If $P-p_{1}>\bar{v}_{2}$ (or $P-p_{2}>\bar{v}_{1}$ ), then $\mu_{12}=0$ because each type prefers buying only object 1 (only object 2) to buying the bundle. However, the profit remains unchanged if $\mathrm{M}$ reduces the price of the bundle to satisfy $P=\min \left\{p_{1}+\bar{v}_{2}, p_{2}+\bar{v}_{1}\right\}$.
} 
and

$$
\begin{aligned}
\frac{\partial \mu_{1}}{\partial P} & =f_{2}\left(P-p_{1}\right)\left[1-F_{1}\left(P-p_{2}\right)\right], \quad \frac{\partial \mu_{2}}{\partial P}=f_{1}\left(P-p_{2}\right)\left[1-F_{2}\left(P-p_{1}\right)\right], \\
\frac{\partial \mu_{12}}{\partial P} & =-f_{1}\left(P-p_{2}\right)\left[1-F_{2}\left(P-p_{1}\right)\right]-f_{2}\left(P-p_{1}\right)\left[1-F_{1}\left(P-p_{2}\right)\right] .
\end{aligned}
$$

Since $\frac{\partial \Pi}{\partial P}=p_{1} \frac{\partial \mu_{1}}{\partial P}+p_{2} \frac{\partial \mu_{2}}{\partial P}+P \frac{\partial \mu_{12}}{\partial P}+\mu_{12}$, after rearranging we obtain $\frac{\partial \Pi}{\partial P}=-\left(P-p_{1}\right) f_{2}\left(P-p_{1}\right)\left[1-F_{1}\left(P-p_{2}\right)\right]+\left[1-F_{1}\left(P-p_{2}\right)-\left(P-p_{2}\right) f_{1}\left(P-p_{2}\right)\right]\left[1-F_{2}\left(P-p_{1}\right)\right]$.

We can argue as in the proof of Proposition 1 to conclude that each term in $\frac{\partial \Pi}{\partial P}$ is negative or zero. Moreover, if $P-p_{2}<\bar{v}_{1}$ then $\frac{\partial \Pi}{\partial P}<0$ since the first term is negative. If $P-p_{2}=\bar{v}_{1}$, then the second term in $\frac{\partial \Pi}{\partial P}$ is $-\bar{v}_{1} f\left(\bar{v}_{1}\right)\left[1-F_{2}\left(P-p_{1}\right)\right]$, which is negative unless $P-p_{1}=\bar{v}_{2}$. In the case that $P=p_{2}+\bar{v}_{1}=p_{1}+\bar{v}_{2}$, we have that $\frac{\partial \Pi}{\partial P}=0$ at $P=p_{2}+\bar{v}_{1}=p_{1}+\bar{v}_{2}$, but $\frac{\partial \Pi}{\partial P}<0$ for $P$ slightly smaller than $p_{2}+\bar{v}_{1}=p_{1}+\bar{v}_{2}$. This makes reducing $P$ strictly profitable.

In the case that $v_{1}, v_{2}$ are i.i.d., we have

$$
\frac{\partial \Pi}{\partial P}=[1-F(P-p)-2(P-p) f(P-p)][1-F(P-p)] .
$$

By the virtue of the same argument used in the proof of Proposition $2, \underline{v}+J^{m} \geq 0$ implies that $1-F(P-p)-2(P-p) f(P-p)<0$, and thus $\frac{\partial \Pi}{\partial P}<0$ if $P-p<\bar{v}$. If instead $P-p=\bar{v}$, then $\frac{\partial \Pi}{\partial P}=0$ for $P=p+\bar{v}$, but $\frac{\partial \Pi}{\partial P}<0$ for $P$ slightly smaller than $p+\bar{v}$.

\subsection{Proof of Proposition 3}

It is convenient to define $\gamma \equiv-u(\underline{v})$, therefore $-u\left(v_{1}\right)$ is equal to $\gamma-\int_{\underline{v}}^{v_{1}} q_{1}(x) d x$, a decreasing function. We use $\pi\left(\gamma, q_{1}\right)$ to denote the profit of $\mathrm{M}$ in (3) as a function of $\left(\gamma, q_{1}\right)$ and prove that $\pi$ is maximized with respect to $q_{1}$ at $q_{1}=q_{1}^{p b}$, with $q_{1}^{p b}$ defined as follows: $q_{1}^{p b}\left(v_{1}\right)=1$ for each $v_{1} \in[\underline{v}, \bar{v}]$. The superscript $p b$ means pure bundling.

The proof is split in three steps. The first two steps establish that $\mathrm{M}$ can restrict his attention to values of $\gamma$ in the interval $[\underline{v}, \bar{v}]$. The third step proves that the optimal $\gamma$ is relatively close to $\underline{v}$, and this implies that the optimal $q_{1}$ is $q_{1}^{p b}$.

Step 1: It is suboptimal to choose $\gamma<\underline{v}$ If $\gamma<\underline{v}$, then $-u\left(v_{1}\right)<v_{1}$ and $F\left(\max \left\{v_{1},-u\left(v_{1}\right)\right\}\right)=F\left(v_{1}\right)$ for any $v_{1} \in[\underline{v}, \bar{v}]$. Hence

$$
\pi\left(\gamma, q_{1}\right)=\int_{\underline{v}}^{\bar{v}}\left[v_{1} q_{1}\left(v_{1}\right)+\gamma-\int_{\underline{v}}^{v_{1}} q_{1}(x) d x\right] f\left(v_{1}\right)\left[1-F\left(v_{1}\right)\right] d v_{1},
$$


which is increasing with respect to $\gamma$. Therefore no $\gamma$ smaller than $\underline{v}$ is optimal.

Step 2: Given $\left(\gamma, q_{1}\right)$ such that $\gamma>\bar{v}$, there exists $\dot{q}_{1}$ such that $\pi\left(\bar{v}, \dot{q}_{1}\right)=\pi\left(\gamma, q_{1}\right)$ First notice that if $-u(\bar{v}) \geq \bar{v}$, then no type participates, that is $F\left(\max \left\{v_{1},-u\left(v_{1}\right)\right\}\right)=1$ for any $v_{1} \in[\underline{v}, \bar{v}]$. Therefore $\pi\left(\gamma, q_{1}\right)=0=\pi\left(\bar{v}, \dot{q}_{1}\right)$ with $\dot{q}_{1}$ such that $\dot{q}_{1}\left(v_{1}\right)=0$ for each $v_{1} \in[\underline{v}, \bar{v}]$.

If $-u(\bar{v})<\bar{v}$, then pick $\dot{v} \in(\underline{v}, \bar{v})$ such that $-u(\dot{v})=\bar{v}$ and let $\dot{q}_{1}\left(v_{1}\right) \equiv\left\{\begin{array}{cc}0 & \text { if } v_{1} \in[\underline{v}, \dot{v}] \\ q_{1}\left(v_{1}\right) & \text { if } v_{1} \in(\dot{v}, \bar{v}]\end{array}\right.$. The equality $\pi\left(\bar{v}, \dot{q}_{1}\right)=\pi\left(\gamma, q_{1}\right)$ holds because the set of participating types and the payment of each participating type are the same in the two cases.

Step 3: If (4) and (5) are satisfied for some $\beta>1$, then $\pi$ is maximized at $\left(\gamma, q_{1}\right)$ such that $q_{1}=q_{1}^{p b}$

The proof of this step is split in three substeps. First we define a function $\tilde{\pi}\left(\gamma, q_{1}\right)$ such that $\pi\left(\gamma, q_{1}\right) \leq \tilde{\pi}\left(\gamma, q_{1}\right)$ and $\pi\left(\gamma, q_{1}^{p b}\right)=\tilde{\pi}\left(\gamma, q_{1}^{p b}\right)$, and then we prove that $\tilde{\pi}$ is maximized with respect to $q_{1}$ at $q_{1}=q_{1}^{p b}$. Since $\pi\left(\gamma, q_{1}\right) \leq \tilde{\pi}\left(\gamma, q_{1}\right)$ and $\pi\left(\gamma, q_{1}^{p b}\right)=\tilde{\pi}\left(\gamma, q_{1}^{p b}\right)$, it follows that also $\pi$ is maximized with respect to $q_{1}$ at $q_{1}=q_{1}^{p b}$.

Step 3.1: The definition of $\tilde{\pi}\left(\gamma, q_{1}\right)$ In view of Steps 1-2, we assume that $\gamma$ belongs to $[\underline{v}, \bar{v}]$, and we let $\hat{v} \in[\underline{v}, \bar{v}]$ be such that $-u\left(v_{1}\right) \geq v_{1}$ for $v_{1} \in[\underline{v}, \hat{v}],-u\left(v_{1}\right)<v_{1}$ for $v_{1} \in(\hat{v}, \bar{v}]$; hence $F\left(\max \left\{v_{1},-u\left(v_{1}\right)\right\}\right)=F\left(-u\left(v_{1}\right)\right)$ if $v_{1} \in[\underline{v}, \hat{v}]$, and $F\left(\max \left\{v_{1},-u\left(v_{1}\right)\right\}\right)=F\left(v_{1}\right)$ if $v_{1} \in(\hat{v}, \bar{v}]$. Therefore

$\pi\left(\gamma, q_{1}\right)=\int_{\underline{v}}^{\hat{v}}\left[v_{1} q_{1}\left(v_{1}\right)-u\left(v_{1}\right)\right] f\left(v_{1}\right)\left[1-F\left(-u\left(v_{1}\right)\right)\right] d v_{1}+\int_{\hat{v}}^{\bar{v}}\left[v_{1} q_{1}\left(v_{1}\right)-u\left(v_{1}\right)\right] f\left(v_{1}\right)\left[1-F\left(v_{1}\right)\right] d v_{1}$.

Let $v^{*} \equiv \frac{1}{2}(\underline{v}+\gamma)$ and notice that $v^{*}=\hat{v}$ if $q_{1}\left(v_{1}\right)=1$ for each $v_{1} \in(\underline{v}, \hat{v}]$, but $v^{*}<\hat{v}$ if $q_{1}\left(v_{1}\right)<1$ for some $v_{1} \in(\underline{v}, \hat{v}]$. A related remark is that $-u\left(v_{1}\right) \geq \gamma-\left(v_{1}-\underline{v}\right)=2 v^{*}-v_{1}$ for $v_{1} \in\left[\underline{v}, v^{*}\right]$, and $-u\left(v_{1}\right) \geq v_{1}$ for $v_{1} \in\left(v^{*}, \hat{v}\right]$. Define $\tilde{\pi}\left(\gamma, q_{1}\right)$ as follows:

$$
\begin{aligned}
\tilde{\pi}\left(\gamma, q_{1}\right) \equiv & \int_{\underline{v}}^{v^{*}}\left[v_{1} q_{1}\left(v_{1}\right)+\gamma-\int_{\underline{v}}^{v_{1}} q_{1}(x) d x\right] f\left(v_{1}\right)\left[1-F\left(2 v^{*}-v_{1}\right)\right] d v_{1} \\
& +\int_{v^{*}}^{\bar{v}}\left[v_{1} q_{1}\left(v_{1}\right)+\gamma-\int_{\underline{v}}^{v^{*}} q_{1}(x) d x-\int_{v^{*}}^{v_{1}} q_{1}(x) d x\right] f\left(v_{1}\right)\left[1-F\left(v_{1}\right)\right] d v_{1} .
\end{aligned}
$$

Since $v_{1} q_{1}\left(v_{1}\right)-u\left(v_{1}\right)=v_{1} q_{1}\left(v_{1}\right)+\gamma-\int_{\underline{v}}^{v_{1}} q_{1}(x) d x>0,{ }^{22}$ we have $\pi\left(\gamma, q_{1}\right) \leq \tilde{\pi}\left(\gamma, q_{1}\right)$.

\footnotetext{
${ }^{22}$ Precisely, $v_{1} q_{1}\left(v_{1}\right)+\gamma-\int_{\underline{v}}^{v_{1}} q_{1}(x) d x=\int_{\underline{v}}^{v_{1}}\left[q_{1}\left(v_{1}\right)-q_{1}(x)\right] d x+\underline{v} q_{1}\left(v_{1}\right)+\gamma$, which is positive since $q_{1}$ is increasing and $\gamma \geq \underline{v}>0$.
} 
Step 3.2: If (4) is satisfied for some $\beta>1$ and $1-\frac{1}{2 \beta}+\frac{1}{2 \beta}\left[F\left(v^{*}\right)\right]^{2} \geq F(\gamma)$, then $\tilde{\pi}$ is maximized with respect to $q_{1}$ at $q_{1}=q_{1}^{p b}$ Consider $q_{1}\left(v_{1}\right)$ for $v_{1} \in\left[v^{*}, \bar{v}\right]$, which affects $\tilde{\pi}$ only through the term

$$
\int_{v^{*}}^{\bar{v}}\left[v_{1} q_{1}\left(v_{1}\right)-\int_{v^{*}}^{v_{1}} q_{1}(x) d x\right] f\left(v_{1}\right)\left[1-F\left(v_{1}\right)\right] d v_{1} .
$$

Integration by parts yields $\int_{v^{*}}^{\bar{v}} \int_{v^{*}}^{v_{1}} q_{1}(x) d x f\left(v_{1}\right)\left[1-F\left(v_{1}\right)\right] d v_{1}=\left[-\frac{1}{2}\left[1-F\left(v_{1}\right)\right]^{2} \int_{v^{*}}^{v_{1}} q_{1}(x) d x\right]_{v^{*}}^{\bar{v}}+$ $\int_{v^{*}}^{\bar{v}} \frac{1}{2}\left[1-F\left(v_{1}\right)\right]^{2} q_{1}\left(v_{1}\right) d v_{1}=\int_{v^{*}}^{\bar{v}} \frac{1}{2}\left[1-F\left(v_{1}\right)\right]^{2} q_{1}\left(v_{1}\right) d v_{1}$, thus (6) is equal to $\int_{v^{*}}^{\bar{v}}\left\{v_{1} f\left(v_{1}\right)[1-\right.$ $\left.\left.F\left(v_{1}\right)\right]-\frac{1}{2}\left[1-F\left(v_{1}\right)\right]^{2}\right\} q_{1}\left(v_{1}\right) d v_{1}$. From (4) it follows that $v_{1} f\left(v_{1}\right)\left[1-F\left(v_{1}\right)\right]-\frac{1}{2}\left[1-F\left(v_{1}\right)\right]^{2} \geq$ $\frac{1}{2}\left(1-F\left(v_{1}\right)\right)\left(2 \beta-1+F\left(v_{1}\right)\right) \geq 0$, and therefore it is optimal to set $q_{1}\left(v_{1}\right)=1$ for any $v_{1} \in\left[v^{*}, \bar{v}\right]$.

Now consider $q_{1}\left(v_{1}\right)$ for $v_{1} \in\left[\underline{v}, v^{*}\right)$, which affects $\tilde{\pi}$ only through the term

$$
\int_{\underline{v}}^{v^{*}}\left[v_{1} q_{1}\left(v_{1}\right)-\int_{\underline{v}}^{v_{1}} q_{1}(x) d x\right] f\left(v_{1}\right)\left[1-F\left(2 v^{*}-v_{1}\right)\right] d v_{1}-\int_{\underline{v}}^{v^{*}} q_{1}(x) d x \frac{\left[1-F\left(v^{*}\right)\right]^{2}}{2} .
$$

Let $\Psi\left(v_{1}\right) \equiv \int_{\underline{v}}^{v_{1}} f(x)\left[1-F\left(2 v^{*}-x\right)\right] d x$, for $v_{1} \in\left[\underline{v}, v^{*}\right]$, and find

$$
\begin{aligned}
\int_{\underline{v}}^{v^{*}} \int_{\underline{v}}^{v_{1}} q_{1}(x) d x f\left(v_{1}\right)\left[1-F\left(2 v^{*}-v_{1}\right)\right] d v_{1} & =\left[\Psi\left(v_{1}\right) \int_{\underline{v}}^{v_{1}} q_{1}(x) d x\right]_{\underline{v}}^{v^{*}}-\int_{\underline{v}}^{v^{*}} \Psi\left(v_{1}\right) q_{1}\left(v_{1}\right) d v_{1} \\
& =\int_{\underline{v}}^{v^{*}}\left[\Psi\left(v^{*}\right)-\Psi\left(v_{1}\right)\right] q_{1}\left(v_{1}\right) d v_{1} \\
& =\int_{\underline{v}}^{v^{*}} \int_{v_{1}}^{v^{*}} f(x)\left[1-F\left(2 v^{*}-x\right)\right] d x q_{1}\left(v_{1}\right) d v_{1} .
\end{aligned}
$$

Therefore (7) is equal to $\int_{\underline{v}}^{v^{*}}\left\{v_{1} f\left(v_{1}\right)\left[1-F\left(2 v^{*}-v_{1}\right)\right]-\int_{v_{1}}^{v^{*}} f(x)\left[1-F\left(2 v^{*}-x\right)\right] d x-\right.$ $\left.\frac{\left[1-F\left(v^{*}\right)\right]^{2}}{2}\right\} q_{1}\left(v_{1}\right) d v_{1}$.

From (4) it follows that

$$
\begin{gathered}
v_{1} f\left(v_{1}\right)\left[1-F\left(2 v^{*}-v_{1}\right)\right]-\int_{v_{1}}^{v^{*}} f(x)\left[1-F\left(2 v^{*}-x\right)\right] d x-\frac{\left[1-F\left(v^{*}\right)\right]^{2}}{2} \\
\geq \beta\left[1-F\left(2 v^{*}-v_{1}\right)\right]-\int_{v_{1}}^{v^{*}} f(x)\left[1-F\left(2 v^{*}-x\right)\right] d x-\frac{1}{2}\left[1-F\left(v^{*}\right)\right]^{2} \equiv \lambda\left(v_{1}\right),
\end{gathered}
$$

and $\lambda$ is an increasing function. Since we have

$$
\lambda(\underline{v})=\beta[1-F(\gamma)]-\int_{\underline{v}}^{v^{*}} f(x)\left[1-F\left(2 v^{*}-x\right)\right] d x-\int_{v^{*}}^{\bar{v}} f(x)[1-F(x)] d x
$$

and

$-\int_{\underline{v}}^{v^{*}} f(x)\left[1-F\left(2 v^{*}-x\right)\right] d x-\int_{v^{*}}^{\bar{v}} f(x)[1-F(x)] d x=-1+\int_{\underline{v}}^{v^{*}} f(x) F\left(2 v^{*}-x\right) d x+\int_{v^{*}}^{\bar{v}} f(x) F(x) d x$ 


$$
\geq-1+\int_{\underline{v}}^{v^{*}} f(x) F\left(v^{*}\right) d x+\frac{1}{2}-\frac{1}{2}\left[F\left(v^{*}\right)\right]^{2}=\frac{1}{2}\left[F\left(v^{*}\right)\right]^{2}-\frac{1}{2},
$$

we infer that

$$
\lambda(\underline{v}) \geq \beta-\frac{1}{2}-\beta F(\gamma)+\frac{1}{2}\left[F\left(v^{*}\right)\right]^{2} \equiv \beta \kappa(\gamma) .
$$

Therefore $q_{1}=q_{1}^{p b}$ maximizes $\tilde{\pi}$ as long as $\kappa(\gamma) \equiv 1-\frac{1}{2 \beta}+\frac{1}{2 \beta}\left[F\left(\frac{1}{2} \underline{v}+\frac{1}{2} \gamma\right)\right]^{2}-F(\gamma)$ is positive or zero. It is immediate that

$$
\kappa(\underline{v})=1-\frac{1}{2 \beta}>0>\kappa(\bar{v})=-\frac{1}{2 \beta}\left\{1-F\left[\left(\frac{1}{2} \underline{v}+\frac{1}{2} \bar{v}\right)\right]^{2}\right\} .
$$

Step 3.3: If (4) and (5) are satisfied for some $\beta>1$, then the optimal $\gamma$ is such that $1-\frac{1}{2 \beta}+\frac{1}{2 \beta}\left[F\left(v^{*}\right)\right]^{2} \geq F(\gamma) \quad$ We prove that if $\gamma$ is such that $\kappa(\gamma)<0$, then $\frac{\partial \tilde{\pi}}{\partial \gamma}<0$. This reveals that the optimal $\gamma$ satisfies $\kappa(\gamma) \geq 0 .{ }^{23}$ After rearranging we find

$$
\begin{aligned}
\frac{\partial \tilde{\pi}}{\partial \gamma} & =\frac{1}{2}\left[1-F\left(v^{*}\right)\right]^{2}-\int_{\underline{v}}^{v^{*}}\left\{\left[v_{1} q_{1}\left(v_{1}\right)+\gamma-\int_{\underline{v}}^{v_{1}} q_{1}(x) d x\right] f\left(2 v^{*}-v_{1}\right)+F\left(2 v^{*}-v_{1}\right)-1\right\} f\left(v_{1}\right) d v_{1} \\
& \leq \frac{1}{2}\left[1-F\left(v^{*}\right)\right]^{2}-\int_{\underline{v}}^{v^{*}}\left[\gamma f\left(2 v^{*}-v_{1}\right)+F\left(2 v^{*}-v_{1}\right)-1\right] f\left(v_{1}\right) d v_{1} .
\end{aligned}
$$

In addition, we have

$$
\begin{gathered}
\int_{\underline{v}}^{v^{*}} \gamma f\left(2 v^{*}-v_{1}\right) f\left(v_{1}\right) d v_{1}>\beta \int_{\underline{v}}^{v^{*}} f\left(2 v^{*}-v_{1}\right) d v_{1} \\
=\beta \int_{v^{*}}^{\gamma} f(x) d x=\beta F(\gamma)-\beta F\left(v^{*}\right)>\beta-\frac{1}{2}-\beta F\left(v^{*}\right)+\frac{1}{2}\left[F\left(v^{*}\right)\right]^{2}
\end{gathered}
$$

where the first inequality follows from (4) and $\gamma>v^{*}$, the first equality is obtained by using the substitution $x=\underline{v}+\gamma-v_{1}$ and the last inequality holds since $\kappa(\gamma)<0$. Moreover, $F\left(2 v^{*}-v_{1}\right) \geq F\left(v^{*}\right)$ for $v_{1} \in\left[\underline{v}, v^{*}\right]$. Therefore

$$
\begin{aligned}
\frac{\partial \tilde{\pi}}{\partial \gamma} & <\frac{1}{2}\left[1-F\left(v^{*}\right)\right]^{2}-\left[\beta-\frac{1}{2}-\beta F\left(v^{*}\right)+\frac{1}{2}\left[F\left(v^{*}\right)\right]^{2}+\left[F\left(v^{*}\right)\right]^{2}-F\left(v^{*}\right)\right] \\
& =-\left(1-F\left(v^{*}\right)\right)\left(\beta-1-F\left(v^{*}\right)\right) .
\end{aligned}
$$

The last expression is negative or zero since $F\left(v^{*}\right) \leq F\left(\frac{1}{2} \underline{v}+\frac{1}{2} \bar{v}\right) \leq \beta-1$ by $(5)$.

\footnotetext{
${ }^{23}$ In order to evaluate $\frac{\partial \tilde{\pi}}{\partial \gamma}$ we use the result that the optimal $q_{1}\left(v_{1}\right)$ is equal to 1 for $v_{1}$ close to $v^{*}$. This follows from the proof of Step 3.2, since $\lambda\left(v_{1}\right)>0$ for $v_{1}$ close to $v^{*}$.
} 


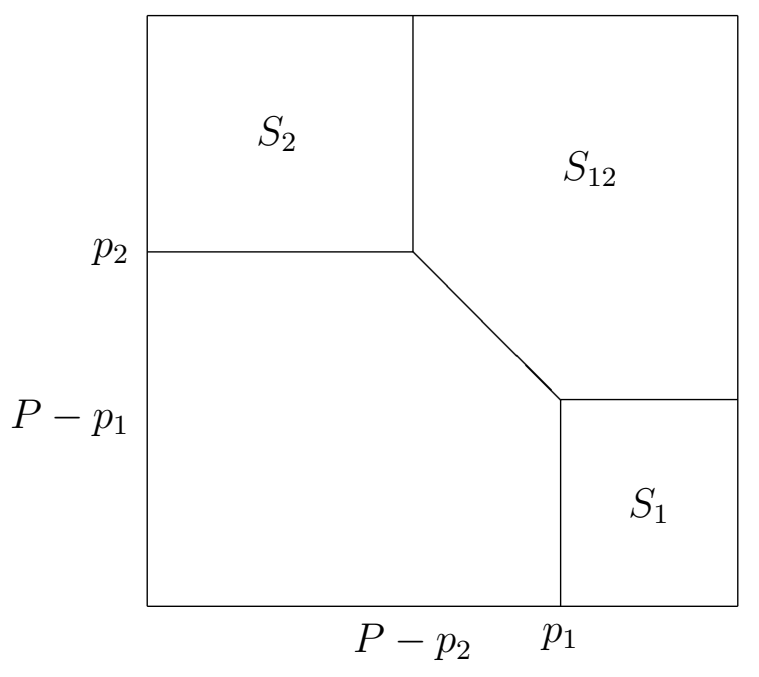

Fig. 1a

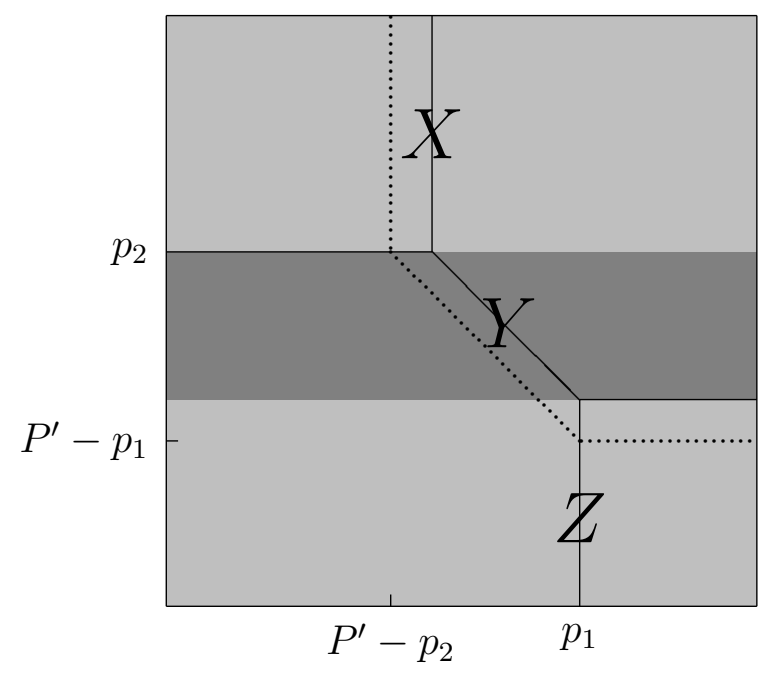

Fig. 1b

Figure 1: Illustration of the proof of Proposition 1. 